\title{
Erratum
}

\section{Life-threatening bleeding from postbulbar duodenal ulcer saved by emergency transcatheter arterial embolization}

Tomotaka Akatsu ${ }^{1}$, Koichi Aiura ${ }^{1}$, Masakazu Ueda $^{1}$, Seishi Nakatsuka ${ }^{2}$, and Masaki Kitajima ${ }^{1}$

${ }^{1}$ Department of Surgery, Keio University School of Medicine, 35 Shinanomachi, Shinjuku-ku, Tokyo 160-8582, Japan

${ }^{2}$ Department of Diagnostic Radiology, Keio University School of Medicine, Tokyo, Japan

J Gastroenterol 2006; 41:604-607

Note

The correct name of the fourth author should be given as Seishi Nakatsuka, not Nakatsuka Seishi. 